\title{
Highly Efficient Nonradiative Energy Transfer from Colloidal Semiconductor Quantum Dots to Wells for Sensitive Noncontact Temperature Probing
}

\author{
Murat Olutas, Burak Guzelturk, Yusuf Kelestemur, Kivanc Gungor, \\ and Hilmi Volkan Demir**
}

This study develops and shows highly efficient exciton-transferring hybrid semiconductor nanocrystal films of mixed dimensionality comprising quasi OD and 2D colloids. Through a systematic study of time-resolved and steadystate photoluminescence spectroscopy as a function of the donor-to-acceptor molar concentration ratio and temperature, a high-efficiency nonradiative energy transfer (NRET) process from CdZnS/ZnS core/shell quantum dots (QDs) directed to atomically flat CdSe nanoplatelets (NPLs) in their solid-state thin films is uncovered. The exciton funneling in this system reaches transfer efficiency levels as high as $\mathbf{9 0 \%}$ at room temperature. In addition, this study finds that with decreasing temperature exciton transfer efficiency is increased to a remarkable maximum level of $\approx 94 \%$. The enhancement in the dipoledipole coupling strength with decreasing temperature is well accounted by increasing photoluminescence quantum yield of the donor and growing spectral overlap between the donor and the acceptor. Furthermore, NRET efficiency exhibits a highly linear monotonic response with changing temperature. This makes the proposed QD-NPL composites appealing for noncontact sensitive temperature probing based on NRET efficiencies as a new metric. These findings indicate that combining colloidal nanocrystals of different dimensionality enables efficient means of temperature probing at an unprecedented sensitivity level at nanoscale through almost complete exciton transfer.

spectral overlap between the donor emission and the acceptor absorption, the fluorescence quantum yield of the donor, the dipole orientation and the refractive index of the medium. ${ }^{[1,2]}$ Although the early applications of FRET were mostly in biology, ${ }^{[3-6]}$ recent studies have shown that FRET can be effectively used for energyefficient optoelectronics ${ }^{[7-21]}$ for enhanced light-generation and -harvesting.

Among semiconductor materials, colloidal semiconductor nanocrystals are interesting candidates for FRET-enabled systems, where FRET is strongly dependent on the dimensionality of the quantum confinement. ${ }^{[22-27]}$ To date, there have been several theoretical and experimental reports on FRET in semiconductor nanocrystals (i.e., colloidal quantum dots (QDs) and nanorods (NRs)) and their hybrids (quantum dot-conjugated polymer, dye, epitaxial quantum well, etc.) regarding the effects of size, composition, dimensionality and architecture on FRET. ${ }^{[21-23,27-31]}$ Such FRETenabled colloidal systems were also shown to be suitable for use in devices including

\section{Introduction}

Förster resonance energy transfer $\left(\right.$ FRET) ${ }^{[1]}$ is a nonradiative energy transfer (NRET) process mediated by near-field dipoledipole coupling between two species (donor and acceptor) that are in close proximity. Exciton transfer rate in FRET depends on the distance between the donor and acceptor species, the light-emitting diodes (LEDs) ${ }^{[20]}$ and solar cells. ${ }^{[32]}$

In recent years, developments in the colloidal synthesis techniques have paved the way for atomically flat quasi-2D NCs, which are referred to as colloidal quantum wells (QWs), or nanoplatelets (NPLs), having a strong 1D confinement in a magic-sized vertical thickness. ${ }^{[33]}$ These colloidal semiconductor NPLs possess unique optical properties with monolayer-level
Dr. M. Olutas, B. Guzelturk, Y. Kelestemur, K. Gungor, Prof. H. V. Demi

Department of Electrical and Electronics Engineering

Department of Physics

UNAM - Institute of Materials Science and Nanotechnology

Bilkent University

Ankara 06800, Turkey

E-mail:volkan@bilkent.edu.tr, hvdemir@ntu.edu.sg

Dr. M. Olutas

Department of Physics

Abant Izzet Baysal University

Bolu 14280, Turkey

\section{Prof. H. V. Demir}

Luminous! Center of Excellence for Semiconductor

Lighting and Displays

School of Electrical and Electronic Engineering

School of Physical and Mathematical Sciences

School of Materials Science and Engineering

Nanyang Technological University

Nanyang Avenue, Singapore 639798, Singapore

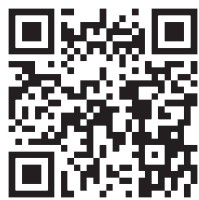

DOI: 10.1002/adfm.201505108 
atomic precision control of their vertical thicknesses. ${ }^{[34]}$ They exhibit extremely narrow photoluminescence (PL) emission $(\approx 30-40 \mathrm{meV}$ at room temperature) as a result of the absence of inhomogeneous broadening. ${ }^{[35,36]}$ Together with giant oscillator strength ${ }^{[34]}$ the large linear and nonlinear absorption cross-sections $^{[37-39]}$ and suppressed Auger recombination ${ }^{[40,41]}$ also make these NPLs attractive candidates for photonic applications, including high-performance colloidal lasers. ${ }^{[36,42,43]}$

Owing to the 2D geometry and extremely large absorption cross-section, the colloidal NPLs stand out as highly efficient exciton acceptors suitable for light-harvesting applications. ${ }^{[44,45]}$ To date, our and Talapin groups' results have shown that ultrafast NRET is possible in the binary colloidal NPLs systems, where excitons are transferred from $4 \mathrm{ML}$ CdSe NPLs to $5 \mathrm{ML}$ CdSe NPLs. ${ }^{[44,45]}$ Furthermore, we have previously shown that an ultraefficient homo-FRET prevails in the stacked assemblies of the same vertical thickness NPLs and this leads to strong quenching of the overall photoluminescence of NPL assemblies due to exciton transport assisted exciton trapping in the defected NPLs. ${ }^{[35]}$

One key advantage of the NPLs that makes them exceptional exciton harvesters is their large absorption cross-section $\left(>10^{-14} \mathrm{~cm}^{2}\right)$. This makes long range dipole-dipole coupling possible with a Förster radius $\geq \approx 10 \mathrm{~nm}$. Recently, 2D monolayers such as graphene and transition metal dichalcogenides (e.g., $\mathrm{MoS}_{2}$ ) have also been shown to exhibit strong exciton sink property through NRET. However, colloidal NPLs with their solution-processability represent a versatile alternative as efficient exciton acceptors for light-harvesting. To date, NRET studies for NPLs have been limited with only NPL based donor-acceptor ensemble systems. ${ }^{[4,45]}$ Even though there are numerous reports on FRET from 3D-confined colloidal semiconductor QDs (as donors) to indirect band-gap semiconductors, ${ }^{[46]}$ 2D materials, ${ }^{[47,48]}$ epitaxial $\mathrm{QWs},{ }^{[49]}$ colloidal $\mathrm{NCs},{ }^{[50]}$ organic dye molecules $^{[23]}$ (as acceptors) and vice versa, ${ }^{[20,22,51,52]}$ FRET has not been studied for an ensemble including colloidal QDs and NPLs. Also, the efficiency limit of such a mixeddimensionality system has not been elucidated to date.

In this work, we report the first study on NRET from colloidal quasi-0D NCs (CdZnS/ZnS core/shell QDs) to quasi2D NCs (CdSe NPLs). Here we systematically investigated the energy transfer processes as a function of the donor-to-acceptor (D/A) molar ratio by using steady-state and time-resolved fluorescence (TRF) spectroscopy in the hybrid films incorporating both the donor QDs and the acceptor NPLs. The significant shortening in PL decay lifetime of the donor QDs and the elongation in the PL decay lifetime of the acceptor NPLs were simultaneously observed, which suggests the exciton transfer from QDs into NPLs. In addition, the existence of NRET was further supported by the PL excitation (PLE) spectra of the acceptor NPLs in the hybrid thin films. We also found that the resulting NRET efficiency can reach unprecedented levels of $\approx 90 \%$ at room temperature owing to the acceptor NPLs. Furthermore, the NRET efficiency further increases up to $\approx 94 \%$ at cryogenic temperatures. The observed enhancement with decreasing temperature is well explained by increasing PL quantum yields and changing in spectral overlaps. We observed that NRET efficiency shows a highly linear monotonic correlation with temperature in the range from 50 to $300 \mathrm{~K}$, which makes this new QD-NPL hybrid system a highly sensitive noncontact optical thermal probe. We also envision that bringing colloidal quantum dots and quantum wells together can lead to a spectrally tunable and efficient exciton harvesting in applications ranging from photovoltaics to photocatalytics.

\section{Results and Discussion}

In this work, we have selected a blue-emitting alloyed-core/ gradient-shell CdZnS/ZnS QDs as an exciton donor due to the fact that they are highly luminescent with long PL decay lifetimes. CdSe NPLs (green-emitting at $\approx 513 \mathrm{~nm}, 4$ monolayer thick) were chosen as an exciton acceptor. Both the donor QDs and the acceptor NPLs were synthesized using modified recipes from the literature. ${ }^{[53,54]}$ The details of the synthesis are described in Experimental Section. Figure 1a,b show the absorption and steady-state PL spectra of the donor and the acceptor materials suspended in hexane at room temperature, respectively. Absorption spectrum of the donor QDs exhibits an absorption onset at $475 \mathrm{~nm}$. The absence of a strong first exciton peak in the absorption is due to alloyed $\mathrm{ZnCdS}$ core, but not due to size distribution. The full-width at half-maximum (FWHM) of the QD emission at the peak emission wavelength (453 nm) is $\approx 30 \mathrm{~nm}$, which is comparable to the best reports for blue-emitting QDs. On the other hand, the acceptor NPLs, thanks to quasi-1D quantum confinement, exhibit epitaxial QW-like absorbance features. The two distinct peaks observed in the NPLs arise from the electron/light-hole (480 nm) and electron/heavy-hole $(512 \mathrm{~nm})$ transitions. In addition, the PL spectrum for the acceptor NPLs shows a single emission peak at $513 \mathrm{~nm}$ with an Fwhm of $\approx 8 \mathrm{~nm}$. All these spectral features consistent with the existing literature suggest that the materials are of high quality. Figure $1 \mathrm{c}, \mathrm{d}$ shows the transmission electron microscopy (TEM) images of both donor and acceptor ensembles on carbon coated copper grids. The mean size of the donor QDs is $9.3 \pm 1.2 \mathrm{~nm}$, whereas that of the acceptor NPLs is $12.8 \pm 1.9$ by $10.5 \pm 1.5 \mathrm{~nm}$.

We prepared seven hybrid thin film samples having different donor and acceptor molar ratios, which is called D/A ratio hereafter, in addition to the reference samples of only donor and only acceptor. The samples were deposited on precleaned quartz substrates using the pre-mixed solutions of the QDs and the NPLs by spin-coating. The molar concentrations of donor (QDs) and acceptor (NPLs) solutions, which were used for the hybrid film preparation process, were calculated as $3.28 \times 10^{-7}$ and $2.16 \times 10^{-6} \mathrm{M}$, respectively. To calculate the molar concentrations, we employed inductively coupled plasma-mass spectrometry (ICP-MS) technique. In addition, the surface coverage of the prepared solid thin film samples were analyzed via scanning electron microscopy (SEM). For an exemplary case, Figure 2 shows the surface coverage of the thin film sample having D/A molar ratio $=0.01$. In Figure $2 \mathrm{a}$ the $\mathrm{QDs}$ are seen as white spots due to their size (with radius $\approx 4 \mathrm{~nm}$ ) creating a larger contrast as compared to the very thin NPLs (vertical thickness $\approx 1.2 \mathrm{~nm}$ ). Although the surface coverage is less than $100 \%$, the QDs and the NPLs are observed to be intermixed well. The donor QDs are well distributed among the acceptor NPLs, which can be found either lying flat or stacked and lying 


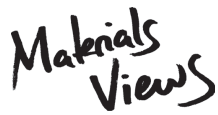

www.MaterialsViews.com

(a)

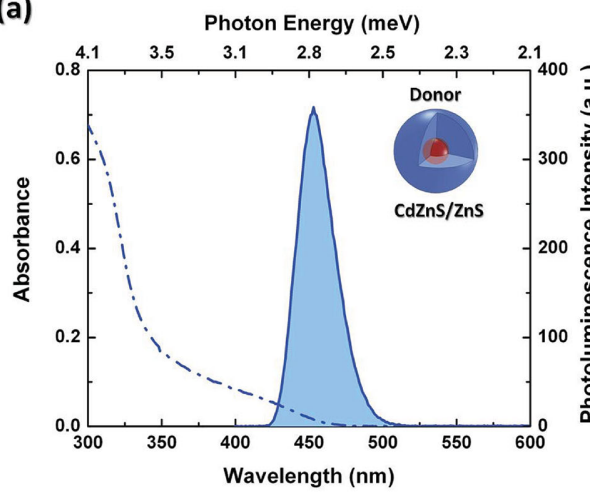

(c)

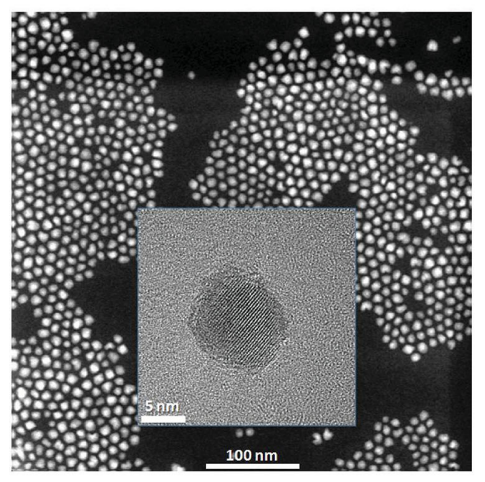

(b)

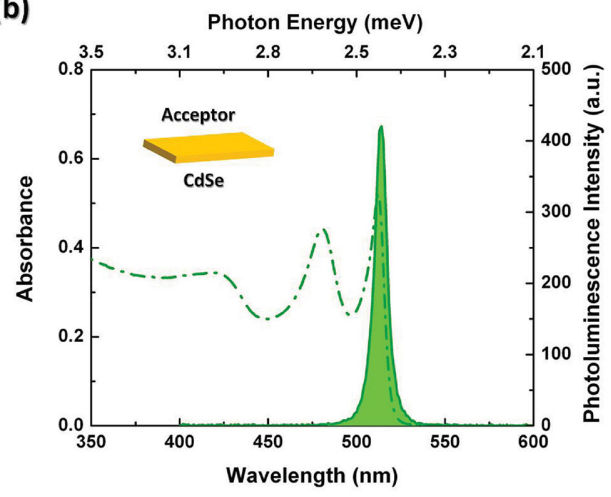

(d)

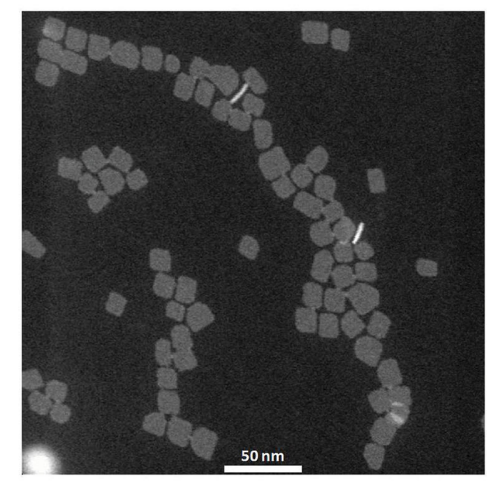

Figure 1. Absorption and photoluminescence spectra of a) the blue-emitting CdZnS/ZnS core/shell QDs and b) the green-emitting 4 ML CdSe NPLs, which are dissolved in hexane at room temperature. High-angle annular dark-field scanning transmission electron microscopy (HAADF-STEM) images of c) the donor-QDs having $9.3 \pm 1.2 \mathrm{~nm}$ and d) the acceptor-NPLs having $12.8 \pm 1.9 \mathrm{~nm}$ by $10.5 \pm 1.5 \mathrm{~nm}$ mean sizes. The inset in (c) shows the TEM image of a CdZnS/ZnS core/shell QDs.

perpendicular to the substrate as shown in Figure $2 \mathrm{~b}$ and cartoon in Figure 2a (see Figures S1-S3 in the Supporting Information for SEM images of different D/A ratios).

Steady-state photoluminescence measurements revealed a evidence of NRET in the hybrid film incorporating both the donor QDs and the acceptor NPLs. Figure 3 shows the PL spectra of the thin films of only-donor, only-acceptor and a hybrid case of with a molar $\mathrm{D} / \mathrm{A}$ ratio of 0.10 , where all samples are excited at $350 \mathrm{~nm}$ by a continuous-wave pump. Hybrid film exhibits strong quenching in donor emission located at $453 \mathrm{~nm}$, while the acceptor emission at $513 \mathrm{~nm}$ is

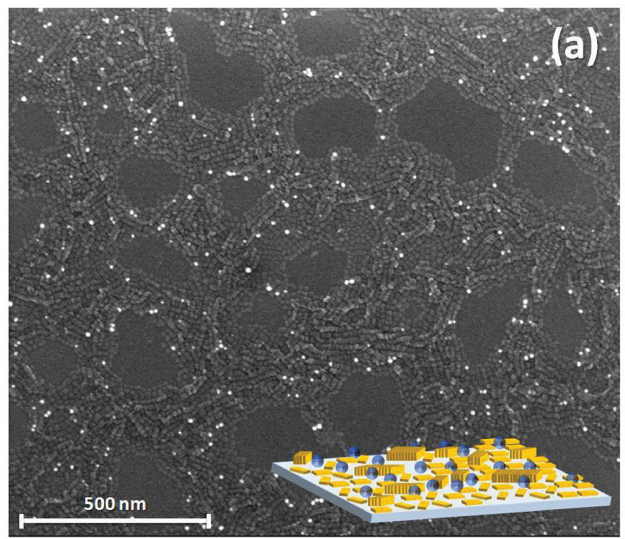

simultaneously enhanced. The quenching ratio of the donor emission suggests $\approx 90 \%$ energy transfer efficiency. However, steady-state PL measurements are not only governed by the NRET, but also affected by the radiative energy transfer due to the absorption of the emitted photons by acceptor NPLs. Therefore, we systematically studied the time-resolved PL kinetics in the hybrid samples with varying D/A ratio, which would directly reveal the NRET efficiencies and its rate.

Next, we performed TRF spectroscopy using a picosecond pulsed pump laser at $375 \mathrm{~nm}$. The decay curves were measured at the band-edge emission peak of both donor $(453 \mathrm{~nm})$

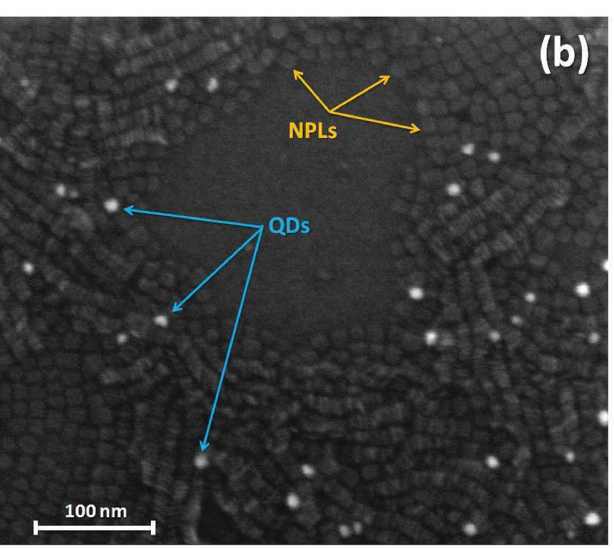

Figure 2. SEM images of the hybrid solid thin films of the donor QDs (bright ones) and the acceptor NPLs having $0.01 \mathrm{D} / \mathrm{A}$ molar ratio for a) $500 \mathrm{~nm}$ and b) $100 \mathrm{~nm}$ scale bars. The yellow and blue arrows given in (b) point at the QDs and the NPLs, respectively. 


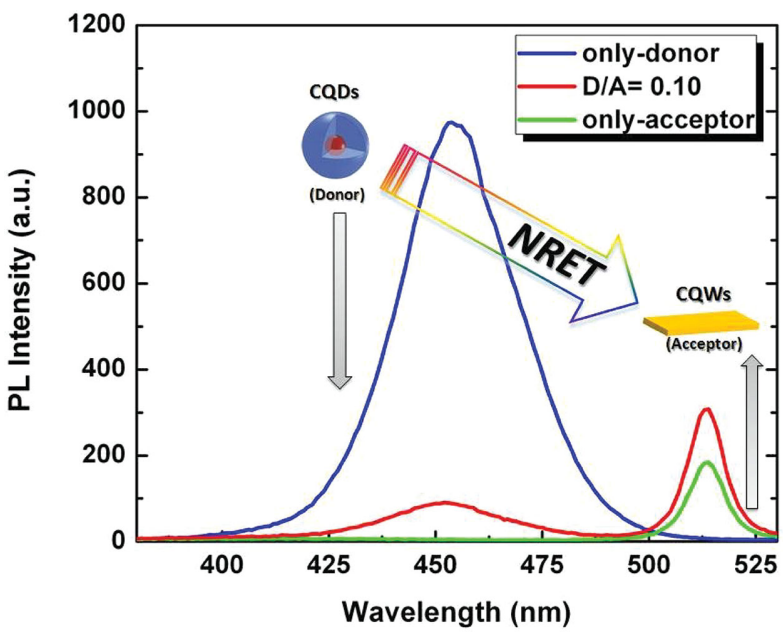

Figure 3. Steady-state PL spectra of the solid films of only-donor (blue line), only-acceptor (green line) and donor-acceptor having $\mathrm{D} / \mathrm{A}=0.10$ (red line) cases.

and acceptor (513 nm) to study NRET at room temperature. There is no cross-talk between the emissions of the donor and acceptor due to narrow emission linewidths of the donor and acceptors. Figure 4 depicts the fluorescence decay curves of prepared samples with different D/A molar ratios. As seen in Figure $4 \mathrm{a}$, the PL decay of the donor QDs becomes faster as the acceptor content of the hybrid film increases. This strongly indicates that an additional relaxation process (e.g., NRET) for the excited state in the donor QDs opens up and it becomes dominant with increasing NPL acceptor concentration in the hybrid films. On the other hand, in the case of the PL decay curves of the acceptor, the decay is observed to slow down with increasing concentration of the QD donors. This elongation in the acceptor decay has been previously observed for the QD, NR, and NPL acceptors, which is the characteristic signature of the delayed luminescence arising from the recombination of the excitons that have been transferred from the donors. Therefore, the changes in the PL decay kinetics of both donor and acceptor indicate the presence of exciton transfer/funneling by NRET. ${ }^{[55]}$

To analyze the PL decay curves, all collected fluorescence decay data were fitted with multi-exponential decay functions. Here, we used four exponential decay functions for both donor and acceptor, which was previously found to be intrinsic for NPLs and this type of QDs. ${ }^{[56,57]}$ The pump laser intensity (at $375 \mathrm{~nm}$, PicoQuant picosecond pulsed laser) was also kept low $\left(\approx 1 \mathrm{~nJ} \mathrm{\textrm {cm } ^ { - 2 }}\right)$ so that number of photogenerated excitons per QD and NPL was much less than $1(\langle\mathrm{~N}\rangle \ll 1)$. The fitting parameters and the amplitude-averaged photoluminescence lifetimes $\left(\tau_{\mathrm{av}}\right)$ of the donor QDs and the acceptor NPLs are summarized in Tables S1 and S2, Supporting Information. $\tau_{\mathrm{av}}$ of the donor QDs decreases from 8.78 (donor-only sample) to $0.92 \mathrm{~ns}$ (sample with $\mathrm{D} / \mathrm{A}=0.01$, where there is the largest number of acceptors per donor). Furthermore, $\tau_{\text {av }}$ of the donor saturates at $\approx 0.90$ ns. These major modifications in the PL lifetime of the donor as a function of the increasing number of acceptor per donor can be explained by the strong energy transfer from the donor QDs to the acceptor NPLs. In the case of acceptors, $\tau_{\mathrm{av}}$ is increased from 0.48 (acceptor-only sample) to $2.19 \mathrm{~ns}$ (sample with $\mathrm{D} / \mathrm{A}=1.37$, where there is the largest number of donors per acceptor).

Subsequently, we analyze the energy transfer rates $\left(k_{\mathrm{NRET}}\right)$ and efficiencies $\left(\eta_{\mathrm{NRET}}\right)$ for all hybrid samples using the fitted PL decay parameters. Table 1 shows the rate of energy transfer as a function of the D/A ratio. As the number of acceptor per donor is increased, the NRET rate increases. The maximum average $k_{\mathrm{NRET}}$ was computed to be $0.97 \mathrm{~ns}^{-1}$. In our analysis

$$
\frac{1}{\tau_{\mathrm{DA}}}=\frac{1}{\tau_{\mathrm{D}}}+k_{\mathrm{NRET}}
$$

was used, where $\tau_{\mathrm{DA}}$ and $\tau_{\mathrm{D}}$ are the amplitude-averaged PL lifetimes of the donor QDs in the presence and absence of acceptor NPLs, respectively. The efficiency of the energy transfer was obtained by the following relation

$\eta_{\mathrm{NRET}}=1-\frac{\tau_{\mathrm{DA}}}{\tau_{\mathrm{D}}}$

Figure 5 depicts $\eta_{\text {NRET }}$ as a function of the varying donor-toacceptor molar concentration ratio. NRET efficiencies progressively increase with the decreasing D/A ratio (see Figure 5). Also, $\eta_{\text {NRET }}$ saturates at $\approx 90 \%$ for the D/A ratios below 0.04 . To the best of our knowledge, the NRET efficiency found here is the highest reported efficiency at room temperature between colloidal based semiconductor NCs in their ensembles. Previously, the NRET efficiency was reported up to $80 \%$ in the case of QD-based donor-acceptor pairs. ${ }^{[12,58]}$ In our previous report using all NPL based donor-acceptor pairs, the NRET efficiency was limited ( $\approx 60 \%)$ in the hybrid solid thin films of 4 and $5 \mathrm{ML}$ NPLs donor-acceptor pairs due to inter-NPL stacking. ${ }^{[4]}$ Thus, the inter-stacking of the each NPL population leads to phase segregation between the donor and acceptor pairs, which considerably increases the donor-acceptor distance and limits the NRET efficiency. Recently, Talapin and co-workers ${ }^{[45]}$ have shown that NRET between 4 and 5 ML NPLs can be as efficient as $\approx 90 \%$. However, this NRET was only limited to donor-acceptor pairs that are face-to-face stacked and only separated by the ligands. As discussed in the latter work, these face-to-face stacked donor-acceptor NPLs are very few in number in the ensemble. Therefore, the ensemble NRET efficiencies were much lower.

Förster radius in the QD-NPL donor-acceptor system was also calculated. To this end, the molar extinction coefficient of the NPL acceptors at $512 \mathrm{~nm}$ was calculated to be $1.46 \times 10^{7} \mathrm{M}^{-1} \mathrm{~cm}^{-1}$, while the quantum yield (QY) of the QD donors in film was determined to be $38 \%$ using an integrating sphere (see Experimental Section for detail). In addition, the refractive index of the medium was taken to be 1.8 , whereas for the dipole orientation factor $\left(\kappa^{2}\right)$ we used $2 / 3$ corresponding to random dipole orientations. Using these parameters given above, the Förster radius was computed to be $9.51 \mathrm{~nm}$. In addition, to estimate the distance between the adjacent QDs and NPLs, we also used the calculated Förster radius and found the closest distance between the QDs and the NPLs to be $5.48 \mathrm{~nm}$ by taking into account the distance scaling of the exciton transfer in our hybrid system is $r^{-4}$, where the $r$ is average distance between donor and acceptor. ${ }^{[26]}$ 

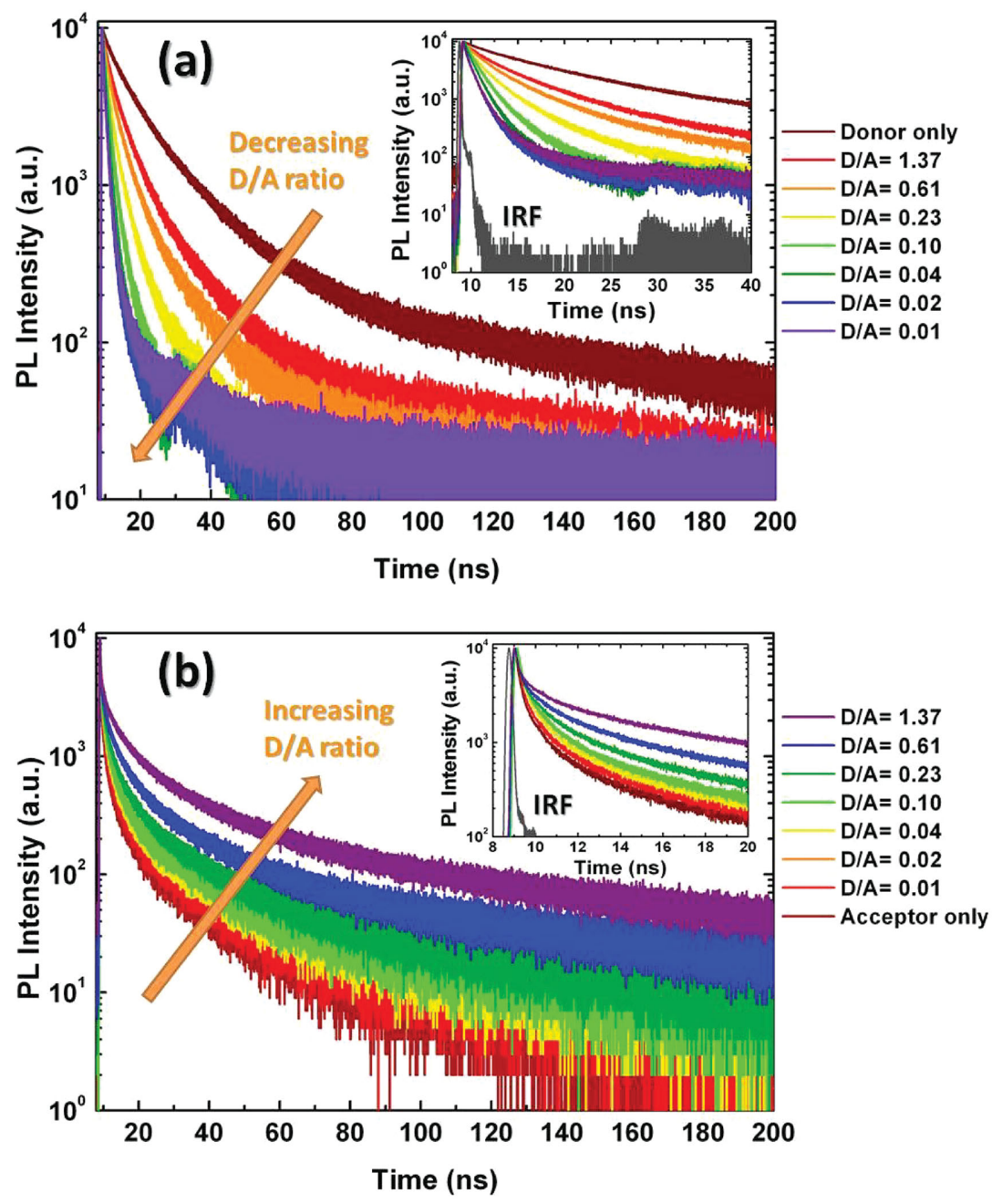

Figure 4. Time-resolved fluorescence decay curves of the solid thin film samples including donor-only, acceptor-only, and hybrid cases with varying D/A molar ratios measured a) at the donor band-edge emission wavelength $(453 \mathrm{~nm})$ and $b$ ) at the acceptor band-edge emission wavelength $(513 \mathrm{~nm})$. The insets show the zoom-in of the same decay curves and the impulse response function (IRF)

To further investigate systematically the steady-state evidence of NRET, PLE spectroscopy was also carried out for

Table 1. Donor QDs and acceptor NPLs lifetimes $(\tau)$, their NRET rates $\left(k_{\text {NRET }}\right)$ and efficiencies $\left(\eta_{\text {NRET }}\right)$ with varying D/A ratios.

\begin{tabular}{lcccc}
\hline D/A ratio & $\begin{array}{c}\tau_{\text {acceptor }} \\
{[\mathrm{ns}]}\end{array}$ & $\begin{array}{c}\tau_{\text {donor }} \\
{[\mathrm{ns}]}\end{array}$ & $\begin{array}{c}k_{\text {NRET }} \\
{\left[\mathrm{ns}^{-1}\right]}\end{array}$ & $\eta_{\text {NRET }}$ \\
\hline Acceptor only & 0.48 & - & - & - \\
0.01 & 0.49 & 0.92 & 0.973 & 0.90 \\
0.02 & 0.48 & 0.92 & 0.973 & 0.90 \\
0.04 & 0.51 & 1.24 & 0.686 & 0.86 \\
0.10 & 0.59 & 1.65 & 0.492 & 0.81 \\
0.23 & 1.00 & 2.26 & 0.329 & 0.74 \\
0.61 & 1.21 & 2.99 & 0.221 & 0.66 \\
1.37 & 2.20 & 4.09 & 0.130 & 0.53 \\
Donor only & - & 8.78 & - & - \\
\hline
\end{tabular}

our donor-acceptor hybrid thin film samples. Figure 6a depicts the PLE spectra of the thin films including the bare donor and acceptor, which were measured for their peak band-edge emission wavelengths. Excitation spectra given in Figure 6a exhibit similar spectral features as the absorption spectra of the QDs and NPLs (see Figure 1). For example, the peak at $480 \mathrm{~nm}$ in the excitation spectrum of the NPLs arises from the electron-light hole (e-lh) transition in the absorption spectrum of acceptor NPLs. We also measured the excitation spectra of the hybrid donor-acceptor cases with varying D/A molar ratios. Figure $6 \mathrm{~b}$ exhibits the excitation spectra of the NPLs (measured at the emission wavelength of $513 \mathrm{~nm}$ ) in the four different hybrid samples with the D/A molar ratios of $0.02,0.10,0.23$, and 0.61 . These excitation spectra are normalized at the e-lh transition peak $(\approx 480 \mathrm{~nm})$ of acceptor since donor QDs do not have any absorption at this wavelength (see Figure 1a). Therefore, Figure $6 \mathrm{~b}$ depicts the normalized excitation spectra of the NPLs. The excitation spectra of the NPLs show increased intensity for the shorter wavelengths with increasing number of donor QDs per NPL (i.e., increasing D/A ratio), which is in alignment with the direction of exciton funneling from the donor to the acceptor. Furthermore, the spectral enhancements in the excitation spectra of the hybrid samples were computed by dividing the normalized excitation spectra of the hybrid cases to that of the acceptor-only case. The inset of Figure $6 \mathrm{~b}$ represents the relative spectral enhancement of the acceptor NPL emission due to the NRET from the donor QDs for given D/A molar ratios. The enhancement of the excitation curve resembles the absorption of the donor QDs. Furthermore, the spectral enhancement is found to reach to twofolds for the sample with large number of donors per acceptor $(\mathrm{D} / \mathrm{A}=0.61)$.

To investigate the effect of temperature on the NRET in the hybrid solid thin films of the donor QDs-acceptor NPLs, we performed time-resolved fluorescence measurements as a function of the temperature under vacuum using a closed cycle $\mathrm{He}$ cryostat. Here, we investigated the thin film sample exhibiting the highest NRET efficiency $(\approx 90 \%)$ at room temperature (RT), which has the lowest D/A molar ratio (i.e., 0.01) in our sample set, together with the donor-only and the acceptor-only samples as reference samples. The measured PL decay curves as a function of the temperature were again fitted by multi-exponential decay functions, and the fitting parameters are given in Tables S3-S6 in the Supporting Information. In the case of the donor-only and the acceptor-only, as seen in Figures S4a and S4b in the Supporting Information, the PL decay curves are accelerated with decreasing temperature from 270 to $50 \mathrm{~K}$. Such a temperature dependent PL lifetime behavior was previously 


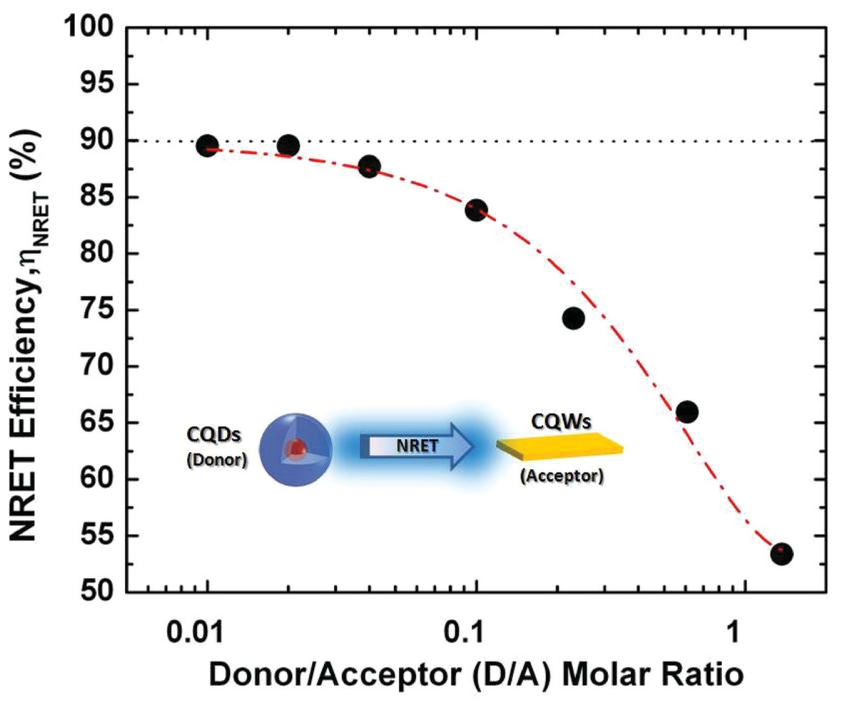

Figure 5. Evolution of the NRET efficiency as a function of the donor QDs to acceptor NPLs (D/A) molar ratios. The dash-dot lines are a guide for the eyes.

reported for NPLS ${ }^{[34]}$ and epitaxial QWs, ${ }^{[59]}$ revealing the giant oscillator strength transition (GOST). The PL lifetime measurements were also carried out for the hybrid sample with D/A molar ratio 0.01, and Figures S4c and S4d in the Supporting Information show the PL decay curves taken at the maximum PL emission peak of both the donor and acceptor as a function of the temperature. Temperature-dependent NRET efficiencies are computed and given in Table S7 in the Supporting Information. Also, Figure 7a provides the NRET efficiency as a function of the temperature. At $270 \mathrm{~K}$, the NRET efficiency is found to be about $87 \%$. Here, the slight decrease in the NRET efficiency, which was measured as $90 \%$ at RT under ambient conditions, can be due the measurement of the decay curves under vacuum conditions. A recent report indicated that the PL lifetime of the NPLs is affected by the oxygen physisorbed at surface of the NPLs. ${ }^{[60]}$ Oxygen passivation of the surface traps elongates the PL lifetime in the NPLs under ambient conditions as compared to the vacuum. Therefore, the change of the PL kinetics of the acceptor is expected to slightly change the overall NRET efficiency under vacuum. As it is seen from Figure 7a, with decreasing temperature, the NRET efficiency increases almost linearly. Also, NRET efficiency remarkably reaches $94 \%$ at $50 \mathrm{~K}$.

To explain the increase in NRET efficiency with decreasing temperature, one possible hypothesis is the enhancement in the spectral overlap between the emission spectrum of the donor and the absorption spectrum of acceptor. To investigate this hypothesis, we first performed the temperature-dependent transmission ( $T$ ) measurements to obtain the absorption behavior of the acceptor with temperature. 1-T (i.e., absorption plus reflection) curves are presented in Figure S5a in the Supporting Information. In addition, the temperature-dependent PL spectra of the donor were taken (see Figure S5b, Supporting Information). We observed that the absorption spectra of the acceptor and the PL spectra of the donor are blue-shifted with decreasing temperature. The calculated spectral shifts $\left(\Delta_{\text {Shift }}\right)$ for given a five set of temperatures (i.e., $270,240,210$, 180 , and $150 \mathrm{~K}$ ) are given in Table S8 in the Supporting Information. Also, we calculated the spectral overlap $(J)$ integrals using the spectral shift differences between the emission spectrum of the donor and absorption spectrum of acceptor with varying temperature (see Table S8 in the Supporting Information). For this, we used the spectral information in Figure S5a. We computed relative enhancement of the NRET efficiencies in the $250-150 \mathrm{~K}$ range and compared them with the experimentally measured NRET efficiencies. Here, we first calculated the relative enhancement of the NRET efficiency by assuming the same parameters (e.g., $\kappa^{2}, n$, QY, and $r$ ) while considering the changing spectral overlap as a function of the temperature. Figure $7 \mathrm{~b}$ shows the computed efficiencies (black diamond) along with the experimental data (blue solid circle). However, the change due to the spectral overlap does not successfully predict the change in the NRET efficiency as a function of the temperature.

Another possible factor for the NRET efficiency is the changing QY of the donor as a function of the temperature. To this end, we also measured the PL QY of the donor for the temperature range under consideration. We found that PL QY of the donor QDs increases from 38\% to $79 \%$ when the temperature is decreased from RT to $150 \mathrm{~K}$ (see Figure S5b and
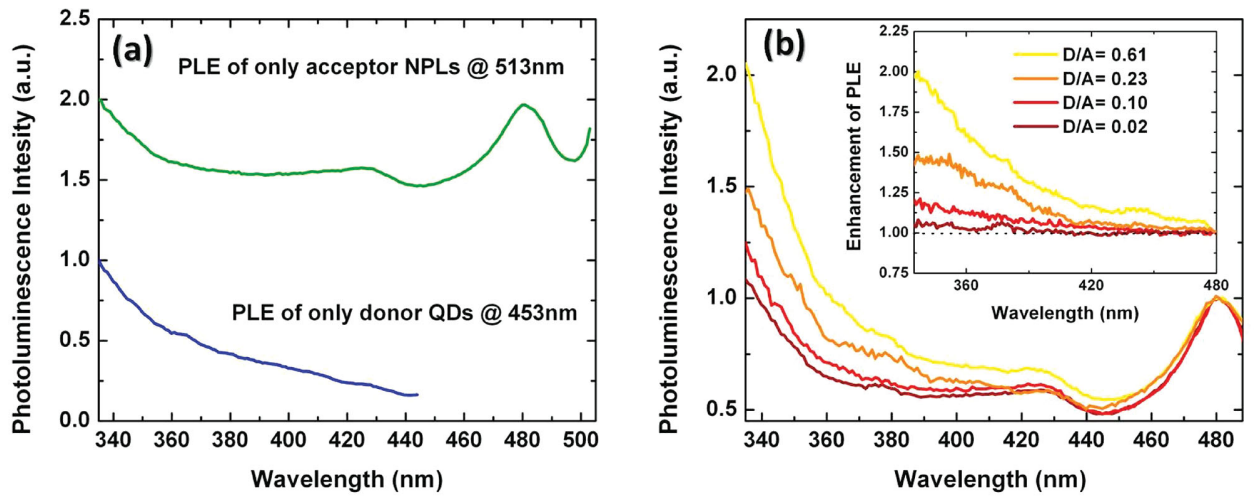

Figure 6. a) PLE spectra of the only-donor QDs (blue-line) and the only-acceptor NPLs (green-line) measured at their emission peak wavelengths (453 and $513 \mathrm{~nm}$, respectively). b) Excitation spectra of the samples having different D/A ratio normalized at the e-lh transition peak wavelength $(480 \mathrm{~nm})$. The inset shows the enhancement in the excitation spectra of the hybrid donor-acceptor samples due to the NRET from donor QDs. 

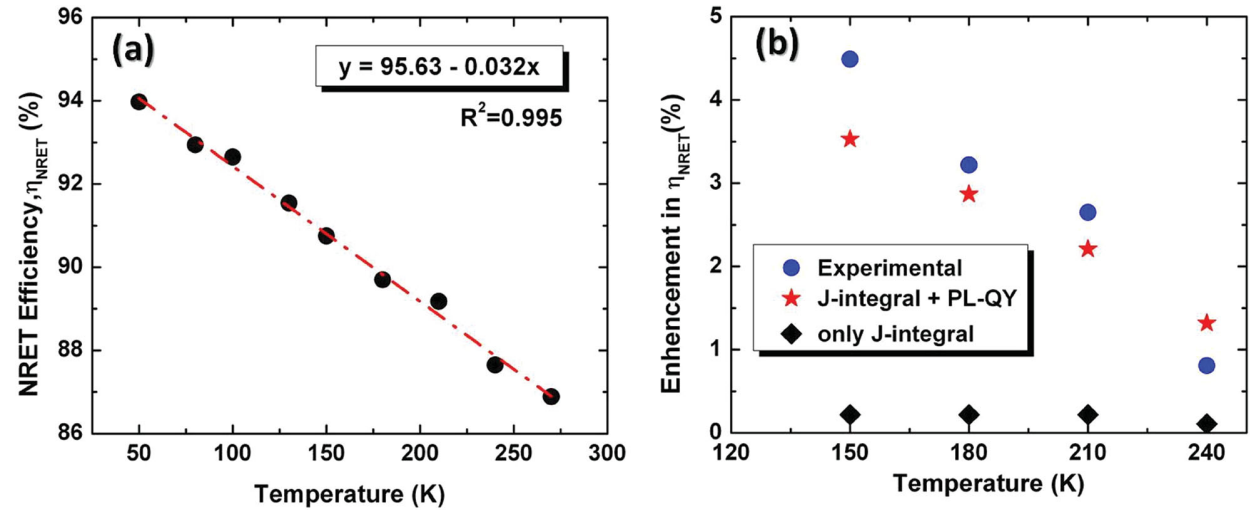

Figure 7. a) Evolution of the NRET efficiency ( $\eta_{\text {NRET }}$ ) for $D / A=0.01$ ratio as a function of the temperature. The dash-dot lines show the linear fitting curve with 0.032 of slope. b) Enhancement in $\eta_{\text {NRET }}$ corresponding based on the experimental data (blue-circle) and the calculated results using the shift in spectral overlap (J-integral) between the PL spectrum of donor and the absorption spectrum of acceptor (black-diamond) and those using both the shift in spectral overlap (J-integral) and the change in PL QY with decreasing temperature.

Table S8 in the Supporting Information). We calculated the relative enhancement of the NRET efficiency (red star) by again assuming the same parameters (e.g., $\kappa^{2}, n$, and $r$ ) while considering the changing quantum yield and spectral overlap as a function of the temperature. The inclusion of the temperature dependent PL QYs resulted in very close agreement with the experimentally observed enhancement of the NRET efficiency over temperature as shown in Figure $7 \mathrm{~b}$. The computed NRET efficiencies $\left(\eta_{\text {NRET-2 }}\right)$ for different temperatures are given in Table S8 in the Supporting Information and the percentage increments in calculated efficiency exhibit very close match with the experimental ones. For example, the percentage enhancements in NRET efficiency $(\Delta-2)$ were calculated to be $1.32 \%, 2.21 \%, 2.87 \%$, and 3.53\% (see the red stars in Figure $7 \mathrm{~b}$ ) corresponding to the NRET efficiency value at $270 \mathrm{~K}$, whereas they were computed to be $0.81 \%, 2.65 \%, 3.22 \%$, and $4.49 \%$ (see the blue solid circles in Figure $7 \mathrm{~b}$ ) for 240, 210, 180, and $150 \mathrm{~K}$, respectively.

In addition, the highly linear correlation between energy transfer efficiency and temperature as shown in Figure 7a highlights the potential of the QD-NPL hybrid system for optical thermal probe applications. Previously, temperature sensing nanocomposites that rely on energy transfer have been studied and proposed. However, all of these previous works have used the change of absolute steady state emission intensities to relate to the temperature change, except the study of Li et al. which was based on linearly temperature dependent fluorescence lifetime of silicon nanoparticles in a limited temperature range of 270-330 K. ${ }^{[21,61-65]}$ Since absolute intensity of the steady state emission is very sensitive to measurement conditions such as sample position, detector and photobleaching, reliability of these previous temperature probing nanocomposites based on ratiometric sensing was quite limited. Here, different from previous reports relying on ratiometric sensing, the presented NRET efficiency represents a new metric for noncontact temperature sensing, which can possibly overcome the reliability limitation of previous nanoscale temperature probes. As shown in Figure 7a, we remarkably achieve a sensitivity level as high as $3.2 \%$ per $\mathrm{K}$ in the broad range of $50-300 \mathrm{~K}$, which is enabled by efficient excition transfer phenomena unique to the QD-NPL hybrids. To the best of our knowledge, our sensitivity level is comparable to the best reported sensitivities (3.5\% per K) in noncontact nanoscale temperature probes. ${ }^{[63,64]}$ In addition to temperature sensing, these nanocomposites with such exceptional exciton transfer efficiencies can further be used in light-harvesting applications in solar cells and artificial photosynthesis.

\section{Conclusion}

In summary, we have investigated the nonradiative energy transfer of excitons in solid thin films of the hybrid ensembles of colloidal CdZnS/ZnS core/shell QDs and CdSe NPLs as a function of the temperature. We found remarkably high NRET efficiencies reaching $90 \%$, which is enabled by large absorption cross-section of the NPLs. To the best of our knowledge, this NRET efficiency level is the highest between colloidal semiconductor NCs reported ever to date. In addition, NRET efficiencies were observed to exhibit highly linear monotonic response and increase with decreasing temperatures, reaching maximum levels as high as $94 \%$, which is well explained by the increasing PL QY of the donor QDs at low temperatures. Owing to the highly linear monotonic behavior of the NRET efficiency with decreasing temperature in the range of 50-300 K, this QD-NPL hybrid system is suitable for the temperature sensing applications with significantly high sensitivity. This presents a new metric based on change of NRET efficiency for temperature sensing, which could possess improved reliability as compared to previously reported nanoscale temperature probes that rely on ratiometric sensing principle. We also believe that such highly efficient NRET between these two colloidal systems having different quantum confinement dimensionality (QDs and NPLs) will enable high-performance optoelectronic devices utilizing FRET at high efficiency.

\section{Experimental Section}

Synthesis of CdZnS/ZnS Core-Shell QDs: $1 \mathrm{mmol}$ of cadmium oxide (CdO), $10 \mathrm{mmol}$ zinc acetate $\left(\mathrm{Cd}(\mathrm{OAc})_{2}\right), 7 \mathrm{~mL}$ of oleic acid $(\mathrm{OA})$, 
and $15 \mathrm{~mL}$ of octadecene (ODE) were loaded to a $100 \mathrm{~mL}$ three-neck flask. The solution was degassed around $100-110^{\circ} \mathrm{C}$ for an hour. After degassing step, the solution was heated to $310^{\circ} \mathrm{C}$ for dissolution of cadmium and zinc precursors. When the precursors were completely dissolved, $2.4 \mathrm{~mL}$ of sulfur stock solution $(0.67 \mathrm{~m} \mathrm{~S}$-ODE solution), which initiated the nucleation of alloyed CdZnS core QDs, was injected into the hot mixture. After the growth of alloyed CdZnS core QDs for $12 \mathrm{~min}, 5 \mathrm{~mL}$ sulfur stock solution $(0.8 \mathrm{~m} \mathrm{~S}$-OA solution) was injected at the rate of $0.5 \mathrm{~mL} \mathrm{~min} \mathrm{~m}^{-1}$ for the successive $\mathrm{ZnS}$ shell coating. After an hour growth at $310^{\circ} \mathrm{C}$, an additional injection of $2 \mathrm{~mL}$ of sulfur stock solution ( $0.8 \mathrm{~m} \mathrm{S-OA}$ solution) was performed with the same rate. Then, the solution was further kept for $2 \mathrm{~h}$ at $310^{\circ} \mathrm{C}$. Finally, the reaction was cooled down to room temperature and $\mathrm{CdZnS/ZnS} \mathrm{core/shell} \mathrm{QDs} \mathrm{were}$ precipitated with the addition of ethanol. Then, the precipitated QDs were dissolved in hexane and stored for further steps.

Synthesis of $4 \mathrm{ML}$ CdSe NPLs: $340 \mathrm{mg}$ of cadmium myristate $\left(\mathrm{Cd}(\mathrm{myr})_{2}\right), 24 \mathrm{mg}$ of selenium (Se) and $30 \mathrm{~mL}$ of ODE were introduced into a $100 \mathrm{~mL}$ three-neck flask. The mixture was degassed under vacuum at $90^{\circ} \mathrm{C}$ with $1000 \mathrm{rpm}$ magnetic stirring until it was completely dissolved and became colorless. After setting the temperature at $240^{\circ} \mathrm{C}$, the vacuum was broken and the flask was filled with argon gas at $100^{\circ} \mathrm{C}$. When the temperature reached $190^{\circ} \mathrm{C}$, the color of solution became yellowish, $120 \mathrm{mg}$ of cadmium acetate dihaydrate $\left(\mathrm{Cd}(\mathrm{OAc})_{2}\left(\mathrm{H}_{2} \mathrm{O}\right)_{2}\right)$ was introduced swiftly into the hot mixture and the reaction was allowed to proceed $10 \mathrm{~min}$ at $240{ }^{\circ} \mathrm{C}$. At the end of the $10 \mathrm{~min}$ growth process, $1 \mathrm{~mL}$ of oleic acid was added and then the mixture was quickly cooled down to room temperature. After adding $5 \mathrm{~mL}$ of hexane into the solution, it was centrifuged for $10 \mathrm{~min}$ at $4500 \mathrm{rpm}$. The supernatant was removed into another centrifuge tube and ethanol was added into supernatant solution untill it became turbid. The turbid solution was centrifuged at $10000 \mathrm{rpm}$ for $10 \mathrm{~min}$, and then the precipitate was dissolved in hexane and filtered with $0.20 \mu \mathrm{m}$ filter.

Preparation of the Solid Thin Films: Using the previously calculated concentrations of $\mathrm{CdZnS} / \mathrm{ZnS}$ QDs $\left(3.28 \times 10^{-7} \mathrm{M}\right)$ and $\mathrm{CdSe}$ $\left(2.16 \times 10^{-6} \mathrm{M}\right) \mathrm{NPLs}$ ensembles dissolved in hexane, the solutions having different $\mathrm{D} / \mathrm{A}$ molar ratios were mixed in glass vials by way of ultrasonication for $5 \mathrm{~min}$. Before using the quartz slides with $1.5 \mathrm{~cm}$ by $1.5 \mathrm{~cm}$ as the substrates for coating, they were cleaned with two steps. First, the quartz substrates were treated with piranha solution and then with deionized (DI) water. In the second step, they were cleaned with acetone and then with isopropanol. After drying the substrates using nitrogen gun, the solid thin films were prepared by spin coating at 2000 rpm for 3 min.

PL QY Measurements: PL QY measurements of the donor-only in film were determined utilizing a Spectral Products monochromator integrated xenon lamp as the excitation source (at an excitation wavelength of $375 \mathrm{~nm}$ ), a Hamamatsu integrating sphere, and an Ocean Optics Maya 2000 spectrometer, and the de Mello method was followed ${ }^{[66]}$ step by step. Briefly, this method basically comprised three different steps. First, spectrum was recorded without sample in the integrating sphere. In the second step, the sample was placed into the integrating sphere and the spectrum was again taken as the sample is directly illuminated by the excitation source. Finally, the sample was rotated and the spectrum was recorded when the sample was excited only under the scattered light from the surface of the integrating sphere. Previously, to test the accuracy of the integrating sphere system, the PL QY of the dye of Rhodamine 6C at $460 \mathrm{~nm}$ excitation wavelength was also measured to be $94.7 \%$, which was in good agreement with the standard value of $95 \%$. In this study, the PL QY of the donor-only in film was measured three times in different time periods as $36.5,38.9$, and $37.6 \%$, and $\approx 38 \%$ of averaged PL QY was used in the calculations.

\section{Supporting Information}

Supporting Information is available from the Wiley Online Library or from the author.

\section{Acknowledgements}

The authors would like to thank for the financial support from Singapore National Research Foundation under the programs of NRFRF-2009-09, NRF-CRP-6-2010-02, and the Science and Engineering Research Council, Agency for Science, Technology and Research (A*STAR) of Singapore (project Nos. 0921010057 and 112120 2009), EU-FP7 Nanophotonics4Energy NoE, and TUBITAK EEEAG 109E002, 109E004, 110E010, 110E217, 112E183, 114E410, and 115E679. H.V.D. acknowledges support from ESF-EURYI and TUBA-GEBIP. The authors would also like to thank Mustafa Guler for his help in TEM images.

Received: November 28, 2015 Revised: January 29, 2016 Published online: March 17, 2016

[1] T. Förster, Ann. Phys. 1948, 437, 55

[2] J. R. Lakowicz, Principles of Fluorescence Spectroscopy, 3rd ed., Springer, New York 2006; pp. 443-468.

[3] X. Qiu, N. Hildebrandt, ACS Nano 2015, 9, 8449.

[4] D. Geissler, S. Linden, K. Liermann, K. D. Wegner, L. J. Charbonniere, N. Hildebrandt, Inorg. Chem. 2014, 53, 1824.

[5] Y. Xiao, F. Shu, K. Y. Wong, Z. Liu, Anal. Chem. 2013, 85, 8493.

[6] A. B. Descalzo, C. Somoza, M. C. Moreno-Bondi, G. Orellana, Anal. Chem. 2013, 85, 5316.

[7] V. G. Kozlov, V. Bulovic, P. E. Burrows, M. Baldo, V. B. Khalfin, G. Parthasarathy, S. R. Forrest, Y. You, M. E. Thompson, J. Appl. Phys. 1998, 84, 4096.

[8] D. J. Farrell, N. J. Ekins-Daukes, Nat. Photonics 2009, 3, 373.

[9] B. Guzelturk, P. L. Hernandez-Martinez, V. K. Sharma, Y. Coskun, V. Ibrahimova, D. Tuncel, A. O. Govorov, X. W. Sun, Q. Xiong, H. V. Demir, Nanoscale 2014, 6, 11387

[10] E. Mutlugun, B. Guzelturk, A. P. Abiyasa, Y. Gao, X. W. Sun, H. V. Demir, J. Phys. Chem. Lett. 2014, 5, 2802.

[11] S. Nizamoglu, B. Guzelturk, D.-W. Jeon, I.-H. Lee, H. V. Demir, Appl. Phys. Lett. 2011, 98, 163108.

[12] N. Cicek, S. Nizamoglu, T. Ozel, E. Mutlugun, D. U. Karatay, V. Lesnyak, T. Otto, N. Gaponik, A. Eychmüller, H. V. Demir, Appl. Phys. Lett. 2009, 94, 061105.

[13] V. Vohra, G. Calzaferri, S. Destri, M. Pasini, W. Porzio, C. Botta, ACS Nano 2010, 4, 1409.

[14] E. Mutlugun, O. Samarskaya, T. Ozel, N. Cicek, N. Gaponik, A. Eychmüller, H. V. Demir, Opt. Express 2010, 18, 10720.

[15] S. Buhbut, S. Itzhakov, E. Tauber, M. Shalom, I. Hod, T. Geiger, K. Y. Garini, D. Oron, A. Zaban, ACS Nano 2010, 4, 1293.

[16] S. Chanyawadee, R. T. Harley, M. Henini, D. V. Talapin, P. G. Lagoudakis, Phys. Rev. Lett. 2009, 102, 077402.

[17] E. Mutlugün, S. Nizamoglu, H. V. Demir, Appl. Phys. Lett. 2009, 95, 033106.

[18] S. Nizamoglu, H. V. Demir, Appl. Phys. Lett. 2009, 95, 151111.

[19] M. Baldo, M. Thompson, S. Forrest, Nature 2000, 403, 750.

[20] M. Achermann, M. a Petruska, S. Kos, D. L. Smith, D. D. Koleske, V. I. Klimov, Nature 2004, 429, 642.

[21] X. Liu, J. Qiu, Chem. Soc. Rev. 2015, 44, 8714.

[22] M. Artemyev, E. Ustinovich, I. Nabiev, J. Am. Chem. Soc. 2009, 131, 8061.

[23] M. Hardzei, M. Artemyev, M. Molinari, M. Troyon, A. Sukhanova, I. Nabiev, ChemPhysChem 2012, 13, 330.

[24] S. Halivni, A. Sitt, I. Hadar, U. Banin, ACS Nano 2012, 6, 2758.

[25] A. Sitt, N. Even-Dar, S. Halivni, A. Faust, L. Yedidya, U. Banin, J. Phys. Chem. C 2013, 117, 22186.

[26] P. L. Hernández-Martínez, A. O. Govorov, H. V. Demir, J. Phys. Chem. C 2013, 117, 10203. 
[27] B. Guzelturk, P. L. H. Martinez, Q. Zhang, Q. Xiong, H. Sun, X. W. Sun, A. O. Govorov, H. V. Demir, Laser Photonics Rev. 2014 $8,73$.

[28] A. L. Rogach, T. A. Klar, J. M. Lupton, A. Meijerink, J. Feldmann, J. Mater. Chem. 2009, 19, 1208.

[29] J. Lee, A. O. Govorov, N. A. Kotov, Nano Lett. 2005, 5, 2063.

[30] J. B. Hoffman, H. Choi, P. V Kamat, J. Phys. Chem. C 2014, 118, 18453.

[31] X. Zhang, C. A. Marocico, M. Lunz, V. A. Gerard, Y. K. Gun'Ko, V. Lesnyak, N. Gaponik, A. S. Susha, A. L. Rogach, A. L. Bradley, ACS Nano 2012, 6, 9283

[32] S. Lu, Z. Lingley, T. Asano, D. Harris, T. Barwicz, S. Guha, A. Madhukar, Nano Lett. 2009, 9, 4548.

[33] S. Ithurria, B. Dubertret, J. Am. Chem. Soc. 2008, 130, 16504

[34] S. Ithurria, M. D. Tessier, B. Mahler, R. P. S. M. Lobo, B. Dubertret, A. L. Efros, Nat. Mater. 2011, 10, 936.

[35] B. Guzelturk, O. Erdem, M. Olutas, Y. Kelestemur, H. V. Demir, ACS Nano 2014, 8, 12524.

[36] C. She, I. Fedin, D. S. Dolzhnikov, A. Demortière, R. D. Schaller, M. Pelton, D. V. Talapin, Nano Lett. 2014, 14, 2772.

[37] A. Yeltik, S. Delikanli, M. Olutas, Y. Kelestemur, B. Guzelturk, H. V. Demir, J. Phys. Chem. C 2015, 119, 26768.

[38] M. Olutas, B. Guzelturk, Y. Kelestemur, A. Yeltik, S. Delikanli, H. V. Demir, ACS Nano 2015, 9, 5041.

[39] A. W. Achtstein, A. Antanovich, A. Prudnikau, R. Scott, U. Woggon, M. Artemyev, J. Phys. Chem. C 2015, 119, 20156.

[40] L. T. Kunneman, M. D. Tessier, H. Heuclin, B. Dubertret, Y. V. Aulin, F. C. Grozema, J. M. Schins, L. D. A. Siebbeles, J. Phys. Chem. Lett. 2013, 4, 3574

[41] E. Baghani, S. K. O. Leary, I. Fedin, D. V Talapin, M. Pelton, J. Phys. Chem. Lett. 2015, 6, 1032.

[42] B. Guzelturk, Y. Kelestemur, M. Olutas, S. Delikanli, H. V. Demir, ACS Nano 2014, 8, 6599.

[43] J. Q. Grim, S. Christodoulou, F. Di Stasio, R. Krahne, R. Cingolani, L. Manna, I. Moreels, Nat. Nanotechnol. 2014, 9, 891.

[44] B. Guzelturk, M. Olutas, S. Delikanli, Y. Kelestemur, O. Erden, H. V. Demir, Nanoscale 2015, 7, 2545.

[45] C. E. Rowland, I. Fedin, H. Zhang, S. K. Gray, A. O. Govorov, D. V. Talapin, R. D. Schaller, Nat. Mater. 2015, 14, 484.

[46] A. Yeltik, B. Guzelturk, P. L. Hernandez-Martinez, A. O. Govorov, H. V. Demir, ACS Nano 2013, 7, 10492.
[47] A. Yeltik, G. Kucukayan-Dogu, B. Guzelturk, S. Fardindoost, Y. Kelestemur, H. V. Demir, J. Phys. Chem. C 2013, 117, 25298.

[48] D. Prasai, A. R. Klots, A. Newaz, J. S. Niezgoda, N. J. Orfield, C. A. Escobar, A. Wynn, A. Efimov, G. K. Jennings, S. J. Rosenthal, K. I. Bolotin, Nano Lett. 2015, 15, 4374.

[49] S. Nizamoglu, E. Sari, J. H. Baek, I. H. Lee, H. V. Demir, Phys. Status Solidi Rapid Res. Lett. 2010, 4, 178

[50] C. Higgins, M. Lunz, A. L. Bradley, V. A. Gerard, S. Byrne, Y. K. Gun'ko, V. Lesnyak, N. Gaponik, Opt. Express 2010, 18, 24486.

[51] S. Nizamoglu, P. Ludwig Hernández-Martínez, E. Mutlugun, D. Ugur Karatay, H. Volkan Demir, Appl. Phys. Lett. 2012, 100, 241109.

[52] B. Guzelturk, H. V. Demir, J. Phys. Chem. Lett. 2015, 6, 2206.

[53] K.-H. Lee, J.-H. Lee, W. Song, H. Ko, C. Lee, J. Lee, H. Yang, ACS Nano 2013, 7, 7295.

[54] M. D. Tessier, P. Spinicelli, D. Dupont, G. Patriarche, S. Ithurria, B. Dubertret, Nano Lett. 2014, 14, 207.

[55] C. Kagan, C. Murray, M. Nirmal, M. Bawendi, Phys. Rev. Lett. 1996 76, 1517

[56] M. D. Tessier, C. Javaux, I. Maksimovic, V. Loriette, B. Dubertret ACS Nano 2012, 6, 6751.

[57] N. Durisic, A. G. Godin, D. Walters, P. Grütter, P. W. Wiseman, C. D. Heyes, ACS Nano 2011, 5, 9062.

[58] T. Franzl, A. Shavel, A. Rogach, N. Gaponik, T. A. Klar, A. Eychmüller, J. Feldmann, Small 2005, 1, 392

[59] J. Feldmann, G. Peter, E. O. Gobel, Phys. Rev. Lett. 1987, 59, 2337.

[60] M. Lorenzon, S. Christodoulou, G. Vaccaro, J. Pedrini, F. Meinardi, I. Moreels, S. Brovelli, Nat. Commun. 2015, 6, 6434.

[61] J. Feng, K. Tian, D. Hu, S. Wang, S. Li, Y. Zeng, Y. Li, G. Yang, Angew. Chem. Int. Ed. 2011, 50, 8072

[62] E. J. McLaurin, V. A. Vlaskin, D. R. Gamelin, J. Am. Chem. Soc. 2011, 133, 14978.

[63] X. Rao, T. Song, J. Gao, Y. Cui, Y. Yang, C. Wu, B. Chen, G. Qian, J. Am. Chem. Soc. 2013, 135, 15559.

[64] S. Zheng, W. Chen, D. Tan, J. Zhou, Q. Guo, W. Jiang, C. Xu, X. Liu, J. Qiu, Nanoscale 2014, 6, 5675.

[65] Q. Li, Y. He, J. Chang, L. Wang, H. Chen, Y. W. Tan, H. Wang Z. Shao, J. Am. Chem. Soc. 2013, 135, 14924.

[66] J. C. de Mello, H. F. Wittmannn, R. H. Friend, Adv. Mater. 1997, 9, 230. 